\title{
Article \\ Genome-Wide Identification of the MYB Gene Family in Cymbidium ensifolium and Its Expression Analysis in Different Flower Colors
}

\author{
Yu-Jie Ke $^{\dagger}$, Qing-Dong Zheng ${ }^{\dagger}$, Ya-He Yao, Yue Ou, Jia-Yi Chen, Meng-Jie Wang, Hui-Ping Lai, Lu Yan, \\ Zhong-Jian Liu * (D) and Ye $\mathrm{Ai}$ *
}

check for

updates

Citation: Ke, Y.-J.; Zheng, Q.-D.; Yao, Y.-H.; Ou, Y.; Chen, J.-Y.; Wang, M.-J.; Lai, H.-P.; Yan, L.; Liu, Z.-J.; Ai, Y. Genome-Wide Identification of the MYB Gene Family in Cymbidium ensifolium and Its Expression Analysis in Different Flower Colors. Int. J. Mol. Sci. 2021, 22, 13245. https://doi.org/ $10.3390 /$ ijms 222413245

Academic Editor: Hikmet Budak

Received: 6 November 2021

Accepted: 5 December 2021

Published: 9 December 2021

Publisher's Note: MDPI stays neutral with regard to jurisdictional claims in published maps and institutional affiliations.

Copyright: (c) 2021 by the authors. Licensee MDPI, Basel, Switzerland. This article is an open access article distributed under the terms and conditions of the Creative Commons Attribution (CC BY) license (https:/ / creativecommons.org/licenses/by/ $4.0 /)$.
Key Laboratory of National Forestry and Grassland Administration for Orchid Conservation and Utilization, College of Landscape Architecture, Fujian Agriculture and Forestry University, Fuzhou 350002, China; 1201775019@fafu.edu.cn (Y.-J.K.); 1191775052@fafu.edu.cn (Q.-D.Z.); ym061866@163.com (Y.-H.Y.); 1201775037@fafu.edu.cn (Y.O.); 1211775002@fafu.edu.cn (J.-Y.C.); wmj281101@fafu.edu.cn (M.-J.W.); lhp990704@fafu.edu.cn (H.-P.L.); yl57@fafu.edu.cn (L.Y.)

* Correspondence: zjliu@fafu.edu.cn (Z.-J.L.); aiye@fafu.edu.cn (Y.A.); Tel.: +86-1362-2392-666 (Z.-J.L.); +86-1856-7910-926 (Y.A.)

+ These authors contributed equally to this work.

\begin{abstract}
MYB transcription factors of plants play important roles in flavonoid synthesis, aroma regulation, floral organ morphogenesis, and responses to biotic and abiotic stresses. Cymbidium ensifolium is a perennial herbaceous plant belonging to Orchidaceae, with special flower colors and high ornamental value. In this study, a total of 136 CeMYB transcription factors were identified from the genome of C. ensifolium, including 27 1R-MYBs, 102 R2R3-MYBs, 2 3R-MYBs, 2 4R-MYBs, and 3 atypical MYBs. Through phylogenetic analysis in combination with MYB in Arabidopsis thaliana, 20 clusters were obtained, indicating that these CeMYBs may have a variety of biological functions. The 136 CeMYBs were distributed on 18 chromosomes, and the conserved domain analysis showed that they harbored typical amino acid sequence repeats. The motif prediction revealed that multiple conserved elements were mostly located in the N-terminal of CeMYBs, suggesting their functions to be relatively conserved. CeMYBs harbored introns ranging from 0 to 13 and contained a large number of stress- and hormone-responsive cis-acting elements in the promoter regions. The subcellular localization prediction demonstrated that most of CeMYBs were positioned in the nucleus. The analysis of the CeMYBs expression based on transcriptome data showed that CeMYB52, and CeMYB104 of the $\mathrm{S} 6$ subfamily may be the key genes leading to flower color variation. The results lay a foundation for the study of MYB transcription factors of $C$. ensifolium and provide valuable information for further investigations of the potential function of MYB genes in the process of anthocyanin biosynthesis.
\end{abstract}

Keywords: Cymbidium ensifolium; MYB transcription factor; anthocyanin; genome

\section{Introduction}

MYB proteins constitute one of the largest transcription factor families in plants [1]. The members of the MYB family have a conserved DNA-binding domain at the Nterminus, which is usually composed of up to four imperfect amino acid repeats harboring 50-53 amino acids [2,3]. According to the number of adjacent repeats, MYB can be divided into four categories, namely 1R-MYB (MYB-related), R2R3-MYB, 3R-MYB (R1R2R3-MYB), and $4 R-M Y B[4,5]$. Among them, the $4 R-M Y B$ and 3R-MYB are only found in a few model plants and little is known about their function [6]. The R2R3-MYB proteins are likely associated with the process of primary and secondary metabolism, plant development, and responses to environmental stresses in plants $[7,8]$.

In addition, numerous studies have shown that R2R3-MYB transcription factors are involved in flavonoids biosynthesis. Subgroup 4 (S4), subgroup (S5), subgroup (S6), and 
subgroup (S7) of the MYB in Arabidopsis thaliana are highly correlated with the flavonoid biosynthetic pathway, among which $\mathrm{S} 4$ is related to the phenylpropane metabolic pathway [9], S5 plays an important role in the proanthocyanidin metabolic pathway, S6 is involved in the anthocyanin biosynthetic pathway (ABP), and S7 participates in the flavonol synthetic pathway [10]. Only S4 acts as a negative regulator while S5, S6, and S7 all serve as positive regulators. Anthocyanin is an important coloring substance in flavonoids, which is closely related to the formation of flower color polymorphisms [11]. ABP is mainly regulated by structural genes and regulatory genes [12]. MBW protein complex, a combination of MYB, bHLH transcription factors, and WD40 proteins, play an important role in regulating the transcription of structural genes [13].

The study of MYB transcription factors is of great significance for flower color polymorphisms of orchids that have special and diverse colors. At present, many MYB genes regulating anthocyanin have been identified in orchids. For example, high levels of anthocyaninspecific MYB transcripts were expressed in the purple but not in the white sectors of petals and sepals of Phalaenopsis 'Everspring Fairy' [14]. The expression of $O g M Y B 1$ is essential to determine the pigment patterns of the floral organs in Oncidium Gower Ramsey [15]. RcPAP1/2 can activate structural genes, which lead to the accumulation of anthocyanin in flowers of $P$. hybrid [16]. The full-red pigment accumulation, the formation of red spots and venation patterns, and other diverse coloring patterns may result from PeMYB2, PeMYB11, and PeMYB12 in the petals of P. equestris [17]. It is worth noting that MYBs also can negatively regulate the accumulation of anthocyanins. $M Y B \times 1$ in $P$. cv. Big Chili [18], PlMYB10 in Pleione limprichtii [19], and MYB10 in Dendrobium phalaenopsis [20] all act as an anthocyanin inhibitor, which reduces the accumulation of anthocyanins by regulating the expression level of structural genes in the ABP.

Cymbidium ensifolium has a long cultivation history and is one of traditional Chinese orchids. It has extremely high ornamental value and is deeply loved by consumers. The color of $C$. ensifolium is various, but its formation mechanism is still unclear. We conducted genome-wide identification of the MYB family members in C. ensifolium by using bioinformatics methods. A total of 136 CeMYB transcription factors were identified, and phylogenetic analysis, chromosomal location, collinearity, gene structure, and conserved motifs were carried out. In addition, we analyzed the expression of CeMYBs in differentcolored sepals of $C$. ensifolium and screened out potential genes that play an important role in flower color polymorphisms. The results of this study lay a foundation for the research of MYB transcription factors in C. ensifolium.

\section{Results}

\subsection{Screened CeMYB Transcription Factors}

A total of 140 CeMYBs transcription factors were initially identified by BLAST searches using whole Arabidopsis MYB protein sequences as the query, and then they were screened out by Pfam. Finally, a total of 136 CeMYBs were obtained in the C. ensifolium genome, which included 27 1R-MYBs, 102 R2R3-MYBs, 2 3R-MYBs, 2 4R-MYBs, and 3 atypical MYBs, were named CeMYB1-CeMYB136 according to their locations on the chromosomes. Further sequence analysis showed that the molecular weight and amino acid (aa) sequence of CeMYB transcription factors were significantly different. The longest CeMYB (CeMYB93) harbored 1106 aa and the shortest (CeMYB76, CeMYB76) were 52 aa. The molecular weights of CeMYBs ranged from 5818.78 to $124,673.79 \mathrm{kDa}$, with isometric points between 10.66 (CeMYB59) and 4.42 (CeMYB28). The main sequence information of CeMYBs is shown in Supplementary Materials Table S1.

\subsection{Phylogeny and Classification of CeMYBs}

The protein sequences of CeMYBs (136) and AtMYBs (131) were used to construct a maximum-likelihood (ML) phylogenetic tree (Figure 1). CeMYBs were divided into 20 subfamilies according to the classification of AtMYBs (Figure 2A) [2]. The S21 subfamily 
harbored the largest number of CeMYBs (a total of 16), followed by S14 (10 members). S7, S9, S16, and S19 had the least CeMYBs (2 members each).

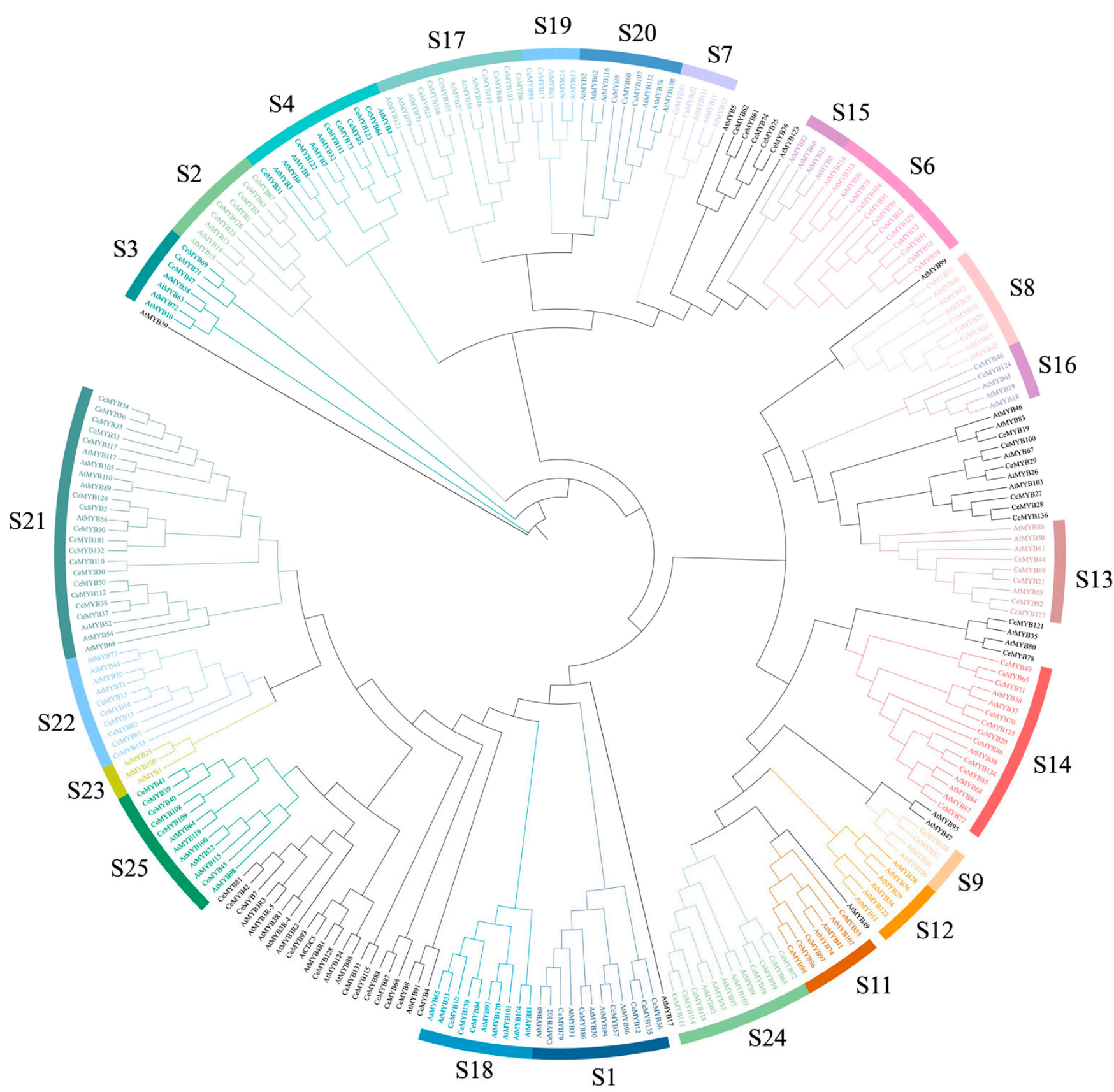

Figure 1. Phylogenetic analysis of MYB proteins from C. ensifolium and A. thaliana. By combining the full-length amino acid sequences of 136 CeMYBs and 131 AtMYBs, a phylogenetic tree was constructed with 1000 bootstrap replicates using IQ-TREE v1.6.12. CeMYBs were divided into subfamilies according to the classification of AtMYBs.

\subsection{Gene Structure and Motif Analysis of CeMYBs}

To investigate the functional diversification of CeMYB proteins, 15 conserved motifs (motifs 1-15) of CeMYB transcription factors were identified through the MEME program. Most motifs were concentrated and regularly distributed at the N-terminus of CeMYBs, and a few motifs were irregularly distributed at the C-terminus. CeMYBs belonging to the same subfamily possessed similar conserved motifs, while different motifs were observed between CeMYBs from different subfamilies. Most CeMYBs had motif 1, 2,3, and 5. Almost all CeMYBs contained more than two conserved motifs, while CeMYB131, CeMYB38, and 
CeMYB40 had only one motif, and CeMYB99, CeMYB83, and CeMYB129 contained motif 1 and motif 5 (Figure 2B). Except that CeMYB86 had motif 10 and CeMYB39 had motif 12, only members of the S1 subfamily had motif 10 and motif 12 . In addition, motif 13 only presented at the C-terminus of the S21 subfamily members. It was speculated that these conserved motifs may be related to the specific function of the subfamily.

A

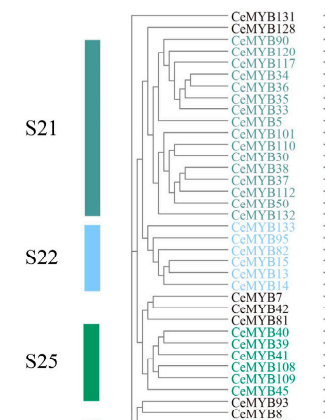

S6

S4

S7

S3

S2

S9

S11

S24

S8

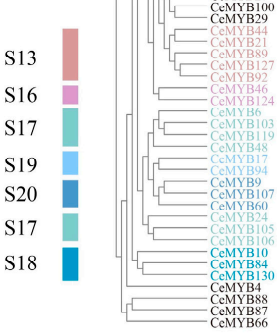

B

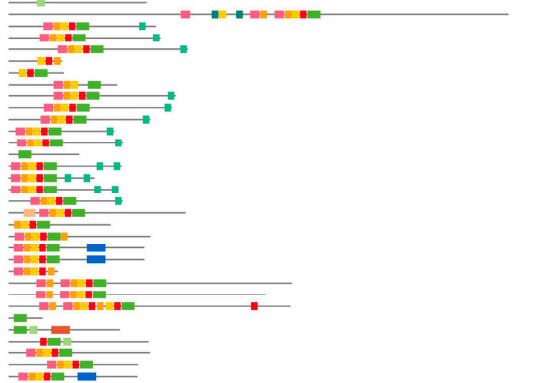

$\mathrm{C}$

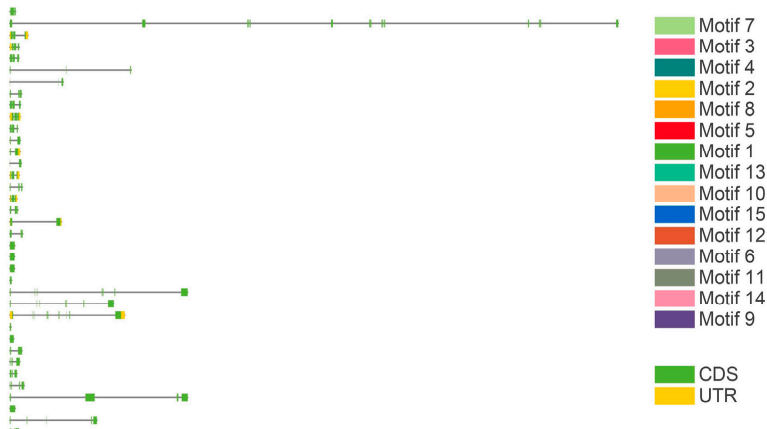

Figure 2. Phylogenetic relationship, conserved protein motif, and gene structure analysis of CeMYBs. (A) A phylogenetic tree harboring 136 CeMYBs. S1 S25 represent subfamilies. (B) Conserved motifs in CeMYBs of different subfamilies. The colored boxes represent the conserved motifs listed on the right side of the figure. Black lines indicate non-conserved sequences. (C) Gene structure of CeMYBs. Green boxes represent exons, black lines connecting two exons represent introns, and yellow boxes represent untranslated regions.

Subsequently, the sequence information of 136 CeMYBs was uploaded to WebLogo to check whether they share conserved sequences. As shown in Figure 3, the R2 and R3 domains of CeMYBs were relatively conserved. About one tryptophan residue in every 18 amino acids was identified. The R2 domain included three tryptophan residues (W) 
(Figure 3A), while the first tryptophan residue in the R3 domain was replaced by phenylalanine residue (F); thus, only two tryptophan residues (Figure $3 \mathrm{~B}$ ) presented in the R3 domain.
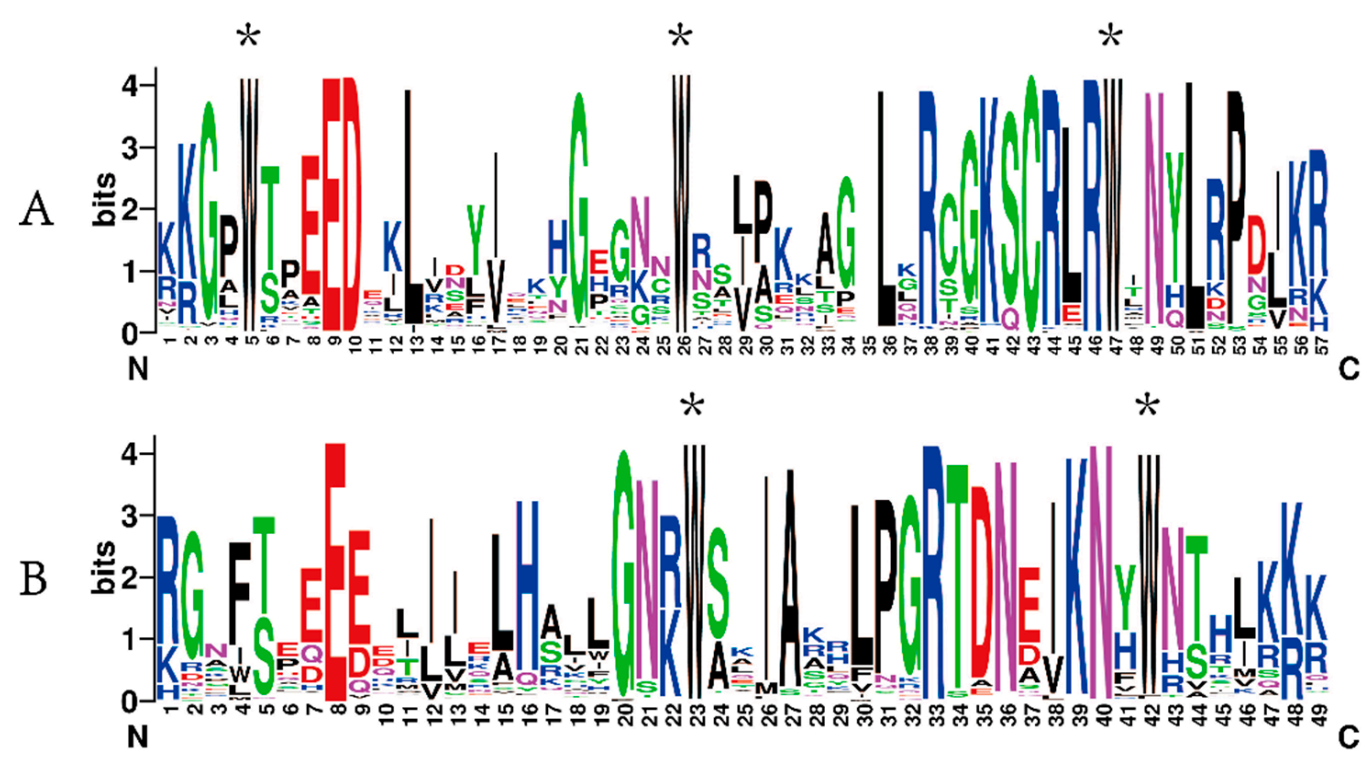

Figure 3. The sequence logos of the R2 (A) and R3 (B) domains. * indicates typical conserved Trp residues in the MYB domain.

As shown in Figure 2C, CeMYB82, CeMYB15, CeMYB13, CeMYB14, CeMYB40,

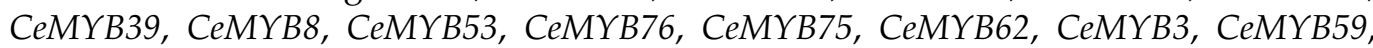
CeMYB115, and CeMYB25 did not have introns, while other CeMYBs possessed introns ranging from 1 to 13 . In total, $66 \mathrm{CeMYBs}$ contained two introns and three exons, accounting for $49 \%$ of the total CeMYBs. These results indicated that although the gene structure of CeMYBs was highly conserved, CeMYBs of different subfamilies showed a high degree of sequence diversity.

\subsection{Analysis of CeMYB Promoters}

The promoters of CeMYBs contained a large number of resistance- and hormoneresponsive cis-acting elements (Supplementary Materials Table S2). Methyl jasmonate (MeJA)-responsive elements (CGTCA motif and TGACG motif) appeared most frequently (a total of 434) in CeMYB promoters, followed by abscisic acid-responsive element (ABRE) and anaerobic induction (ARE) (Figure 4). It is worth noting that there were 10 cis-acting elements (MBSI) regulating flavonoids biosynthesis in CeMYB19, CeMYB85, CeMYB7, CeMYB67, CeMYB30, CeMYB79, CeMYB80, and CeMYB1, indicating that these genes may be involved in the process of flavonoids biosynthesis. The above results indicated that the transcriptional regulation of $C e M Y B s$ was different, suggesting the functions of $C e M Y B$ were diversity.

\subsection{Chromosomal Localization and Collinearity Analysis of CeMYBs}

The chromosome mapping results showed that among 136 CeMYBs, 134 were unevenly distributed on the 18 chromosomes of C. ensifolium, and 2 CeMYBs were localized to the unanchored scaffolds (Figure 5). Chromosome 3 had the most CeMYBs (18); chromosome 7 had 11 CeMYBs; chromosomes 1, 2, and 4 had 10 CeMYBs, respectively; and the other chromosomes contained 1-9 CeMYBs. In addition, the protein subcellular localization results showed that most CeMYBs were localized in the nucleus (Supplementary Materials Table S1). 

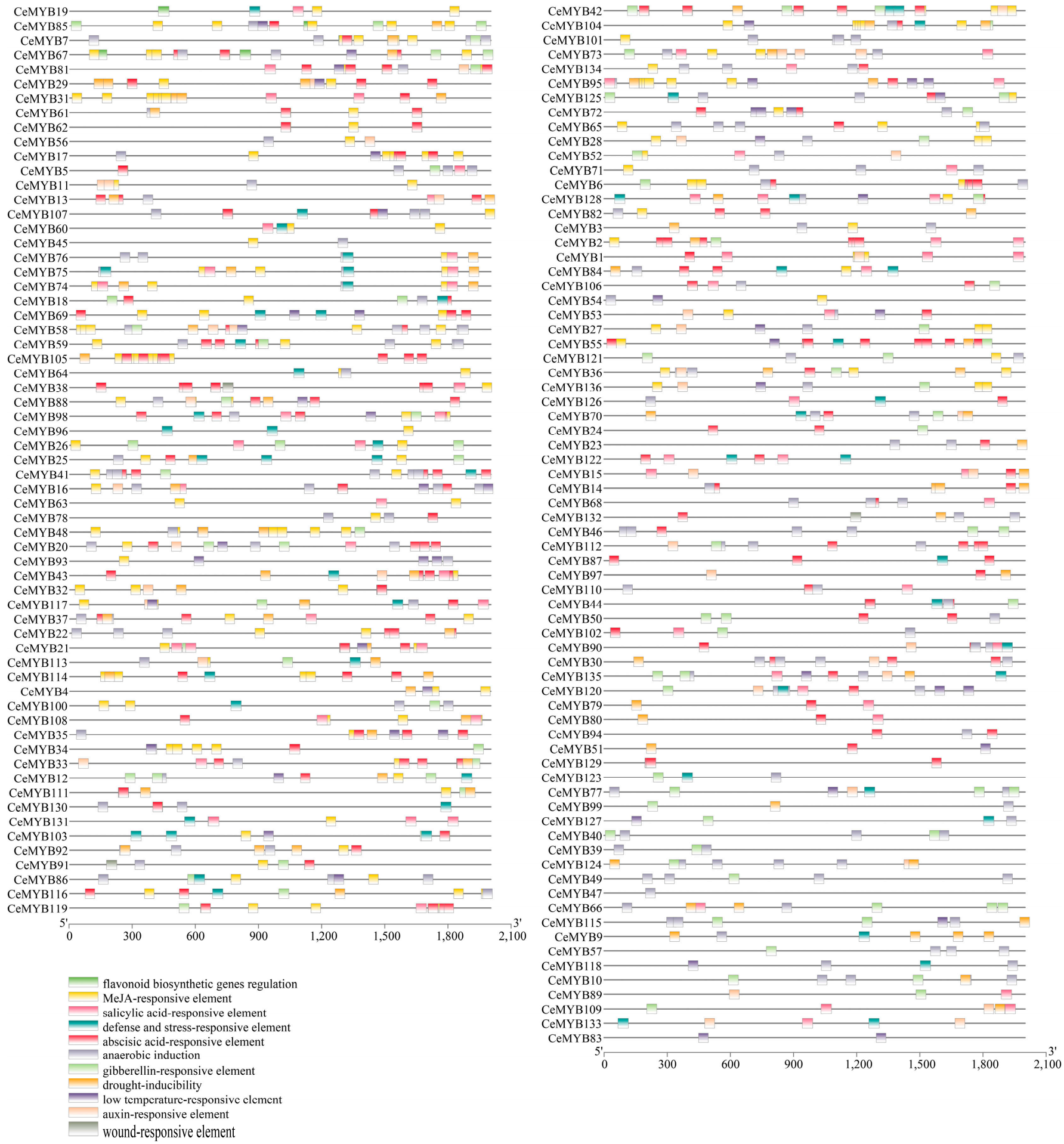

Figure 4. Regulatory elements in the promoter region of CeMYBs as predicted with PlantCARE. Cis-acting elements with similar functions were displayed in the same color. Different colored boxes represent different cis-acting elements.

Both tandem duplication and segmental duplication are conducive to the expansion of gene families in plant [21]. The collinearity analysis revealed that the C. ensifolium genome contained 29 pairs of segmentally duplicated genes (Figure 6). The largest number of segmentally duplicated genes were found on chromosome 12, and only one was found on chromosomes 11, 13,14, 18, and 20, respectively. In addition, 23 tandemly duplicated gene pairs were identified, and most of them occurred on chromosome 3 . Among the tandemly duplicated genes, CeMYB13 and CeMYB15; CeMYB75 and CeMYB76; CeMYB96 
and CeMYB97; CeMYB113 and CeMYB114; CeMYB105 and CeMYB106; and CeMYB87 and $C e M Y B 88$ were located in the approximate position of chromosomes and shared the same conserved motifs and similar gene structure.

The $\mathrm{Ka} / \mathrm{Ks}$ values of six CeMYB gene pairs, which were $\mathrm{NaN}$ (Not a Number), were excluded. The other $46 \mathrm{Ka} / \mathrm{Ks}$ values were less than 1 and $73.9 \%$ of them were lower than 0.5 , suggesting that these genes evolved under the influence of purifying selection. The $\mathrm{Ka} / \mathrm{Ks}$ value of tandemly duplicated genes ranged between 0.18 and 0.82 , while that of segmentally duplicated genes ranged from $0.1-0.44$. The average $\mathrm{Ka} / \mathrm{Ks}$ value of tandemly replicated genes (0.53) was higher than that of segmentally duplicated genes (0.24) (Supplementary Materials Table S3).
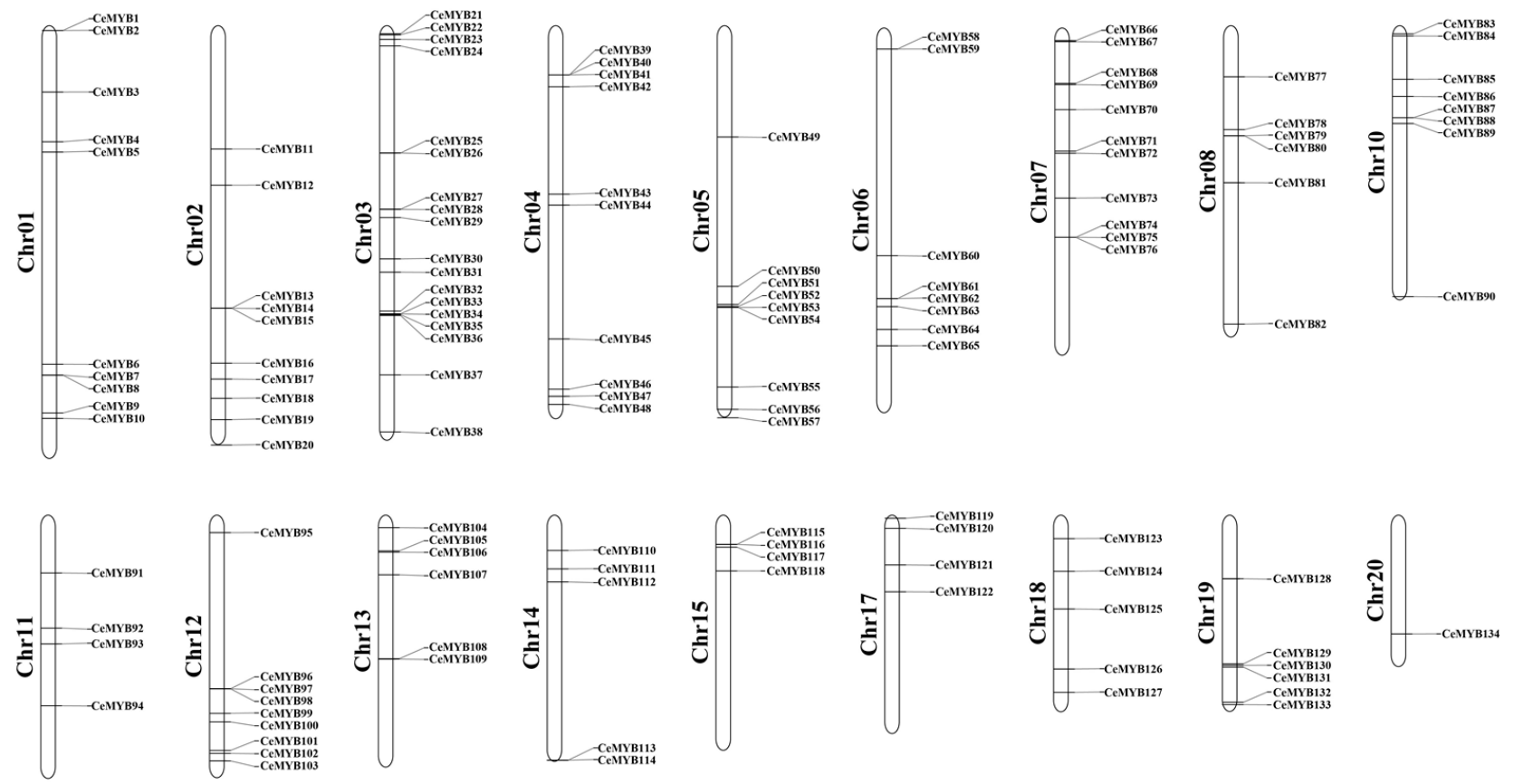

Figure 5. Chromosomal localization analysis of the CeMYBs. The chromosome number is indicated to the left of each chromosome. The scale is in mega bases $(\mathrm{Mb})$. Each gene is named according to the order of its corresponding location on the chromosome.

\subsection{Expression patterns of CeMYBs in Different-Colored Sepals of C. ensifolium}

The expression levels of all CeMYBs in C. ensifolium with different-colored sepals were obtained from the transcriptome data (Supplementary Materials Table S4). Approximately $20.6 \%$ of CeMYBs were not expressed in the sepals of C. ensifolium. A cluster heat map illustrating the expression levels of $108 \mathrm{CeMYBs}$ was created, and these genes were divided into seven groups (A-G) (Figure 7). Among the 108 CeMYBs, 18 genes of group A had low expression levels in white and yellow-green sepals, and high expression levels in red and purple-red sepals. Moreover, the expression pattern of group A genes was consistent with the anthocyanin content in sepals, indicating that the group A genes may be involved in the regulation of anthocyanins. 14 genes in group B were only expressed in purple-red sepals, while low expression levels were shown in sepals with other colors. The expression levels of nine genes in group $C$ were higher in white and purple-red sepals. Among the 22 genes in group D, CeMYB78, CeMYB7, CeMYB132, and CeMYB84 were highly expressed in yellow-green and white sepals, and the other genes were highly expressed in purple-red 
and yellow-green sepals. 15 genes in group E were highly expressed only in white sepals, and 15 genes in group F were highly expressed in red sepals, except CeMYB17. All the genes in group $\mathrm{G}$ were highly expressed in yellow-green sepals, and some genes were also highly expressed in red sepals. It is noteworthy that CeMYB104 and CeMYB52 in group A both belonged to the $\mathrm{S} 6$ subfamily, which can regulate the synthesis of anthocyanins, and should be emphasized in the subsequent studies.

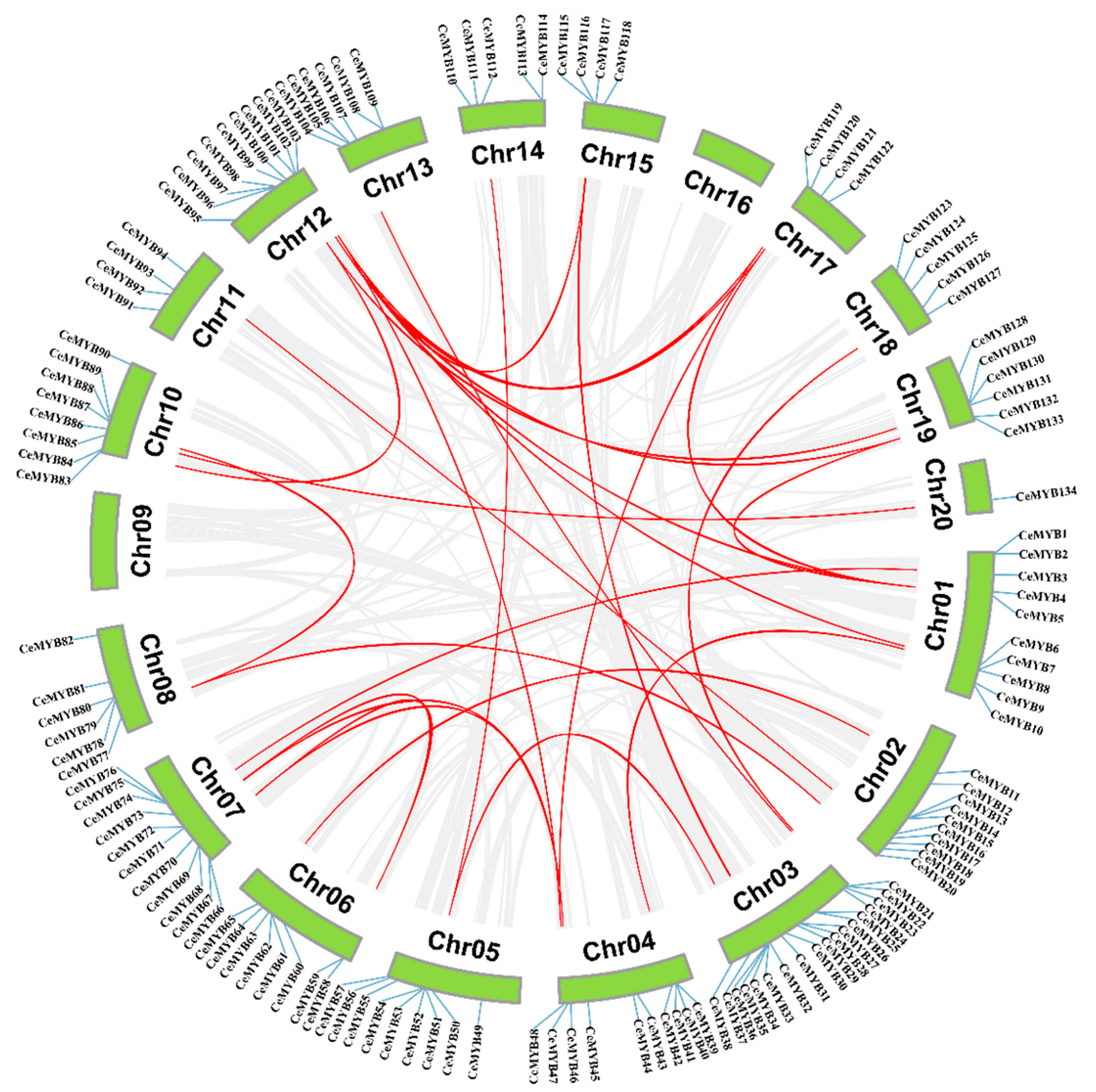

Figure 6. Synteny analysis of CeMYBs in C. ensifolium. Red lines represent the duplicated CeMYB gene pairs in the genome. The chromosome number is displayed next to each chromosome.

\section{7. qRT-PCR Analysis of CeMYBs}

CeMYB52 and CeMYB104 of the S6 subfamily, which are involved in anthocyanin biosynthesis, were selected, and their expression levels in the different-colored petals were investigated (Figure 8). The qRT-PCR results showed that CeMYB52 had the highest expression in purple-red sepals, followed by red sepals, and low expression in yellowgreen and white sepals. CeMYB104 was expressed in purple-red and red sepals but not in white and yellow-green sepals. The expression patterns of CeMYB52 and CeMYB104 in different-colored sepals was basically consistent with the transcriptome sequencing results, which further confirmed the reliability of the transcriptome data. 


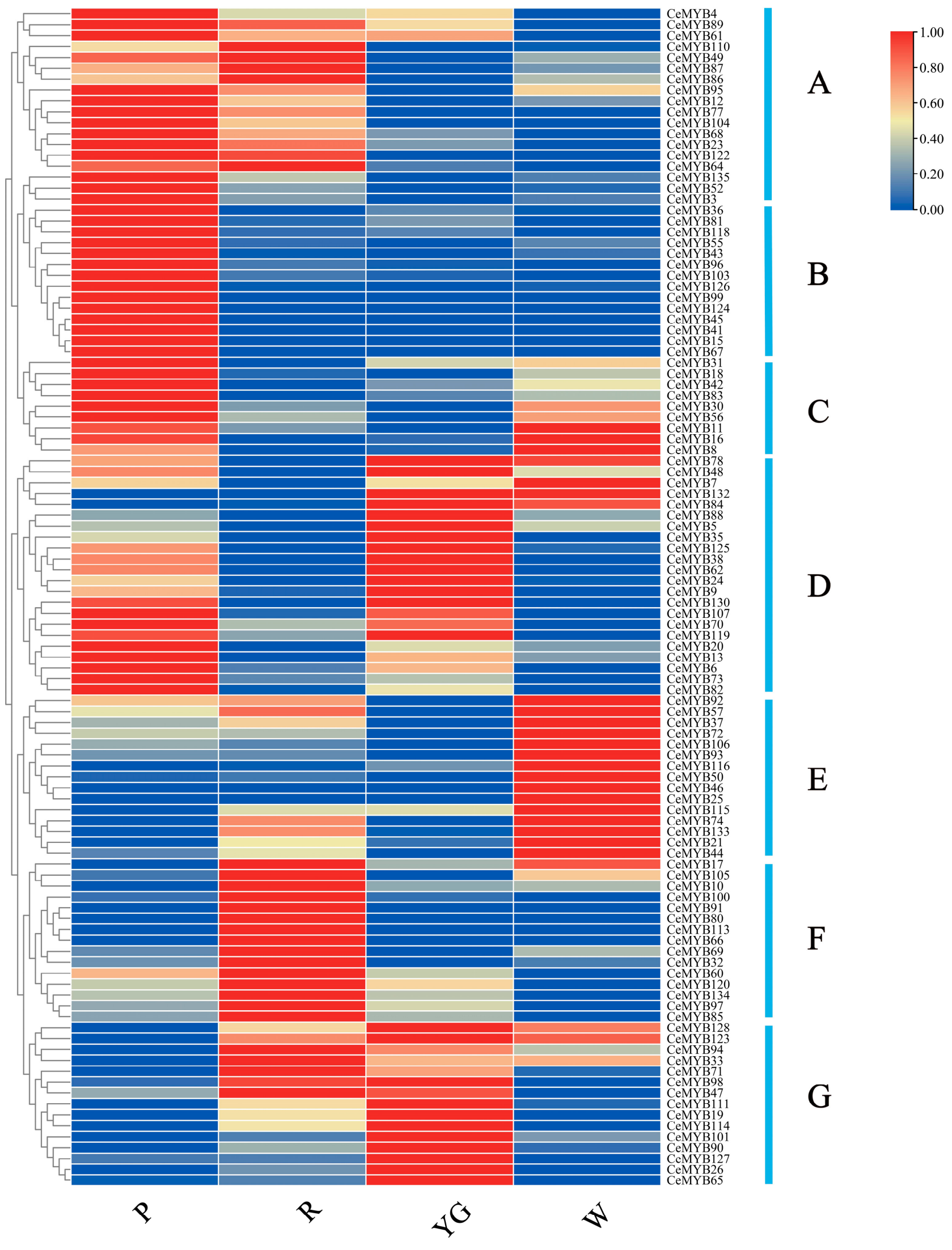

Figure 7. Expression patterns of $108 \mathrm{CeMYBs}$ categorized into seven groups (A-G) according to the expression levels in different sepals. P: purple-red sepals; R: red sepals; YG: yellow-green sepals; W: white sepals. 


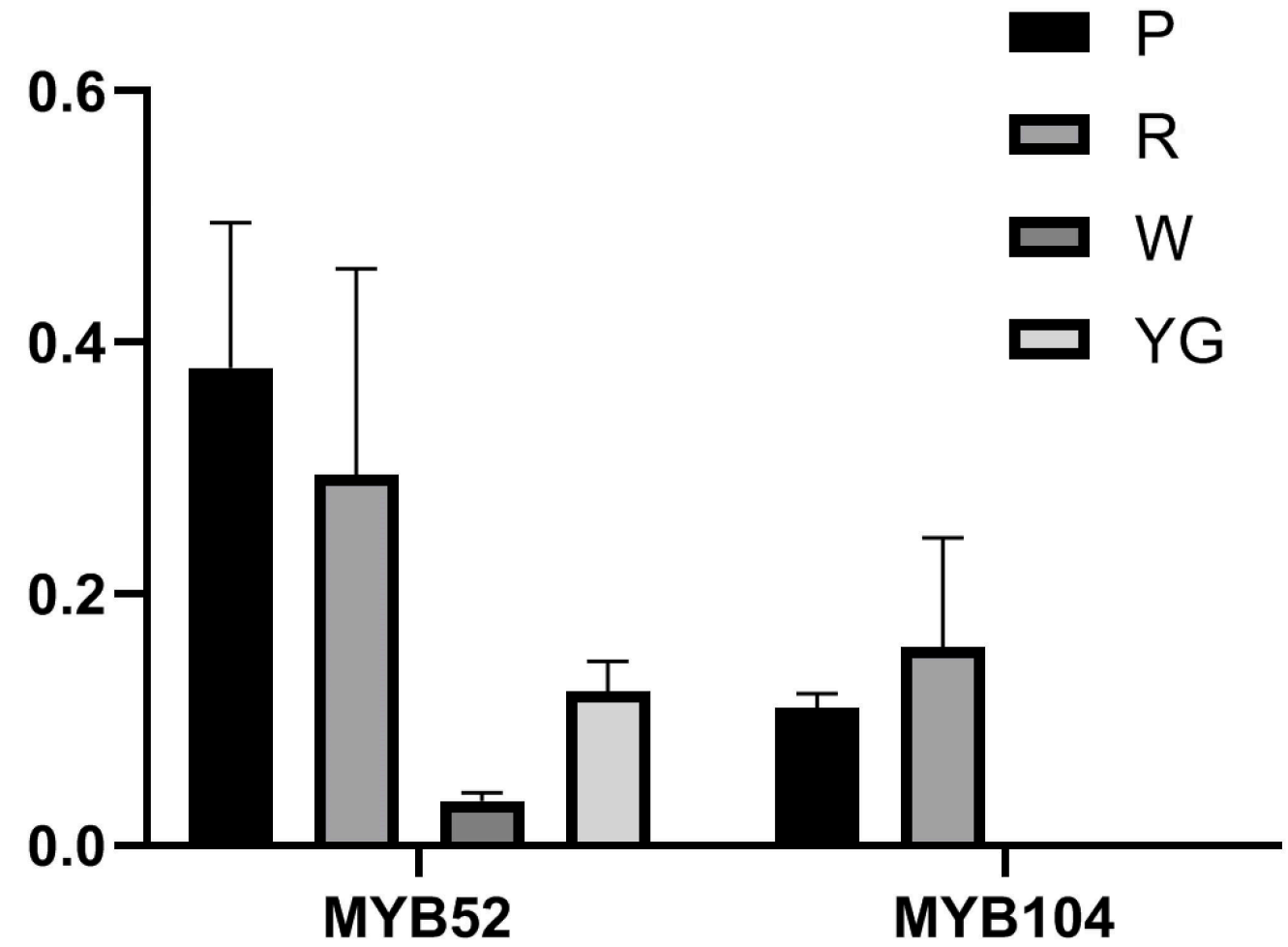

Figure 8. Expression verification of MYB52 and MYB104 in different-colored sepals of C. ensifolium by qRT-PCR. Each value is shown as average \pm standard deviation from three biological replicate sampling. P: purple-red sepals; R: red sepals; YG: yellow-green sepals; W: white sepals.

\section{Discussion}

C. ensifolium, which belongs to the Orchidaceae, is one of the most critical ornamental flowers in China, with diverse colors, elegant shape, and fragrant aroma [22]. In ornamental plants, MYB transcription factors correlate with anthocyanin biosynthesis, flower development, and scent emission [22]. Genome-wide analysis is an essential method for clarifying the biological functions of the MYB family in plants. In this work, 136 CeMYB transcription factors were identified through the $C$. ensifolium genome, with 102 R2R3MYBs, which play an important role in the process of growth and development in plants. The number of R2R3-MYBs in C. ensifolium is similar to P. equestris (96) [23], P. aphrodite (99) [24], D. officinale (101) [24], and have a large gap with other species, such as Glycine max (244) [25] and Triticum aestivum (393) [26]. The reason for the quantitative difference may be caused by the genome size and duplication events, which also reflects the complexity and diversity of plant R2R3-MYB transcription factors in the process of evolution.

Through a phylogenetic analysis combining the MYB proteins from $A$. thaliana, CeMYBs were divided into 20 subfamilies. Owing to the conservation of MYB, these genes with similar or identical functions were classified into the same subgroup. CeMYB52 and CeMYB104 were clustered with AtMYB75 (PAP1), AtMYB90 (PAP2), AtMYB113, and AtMYB114 in the S6, indicating that they were involved in the ABP and were verified in subsequent qRT-PCR experiments. The function of MYB genes still needs to be further explored in different subgroups of C. ensifolium. Moreover, some CeMYBs were not included in subfamilies of $A$. thaliana, suggesting that these genes may be formed after the divergence of $C$. ensifolium from $A$. thaliana and have unique biological functions. Alternatively, these genes might be lost from the $A$. thaliana genome during evolution [27]. The clustering results of CeMYBs and AtMYBs were similar to those of D. officinale, P. aphrodite, and A. thaliana, in which most members were found in the S21 subfamily, and MYB were not classified into the S12 and S15 subfamilies [24], indicating that MYB may not be involved in regulating glucosinolate biosynthesis and root hair patterning in orchids $[28,29]$. This result indicates that the classification of MYB genes in orchids is similar. 
Tryptophan residues in each $\mathrm{R}$ motif contribute to the maintenance of the helix-turnhelix (HTH) structure, enabling the binding of MYB transcription factors to DNA [30]. Like in most other plants, the structural domain of CeMYBs also contained representative tryptophan residues, among which R2 had three highly conserved tryptophan residues, while the first tryptophan residue in R3 was replaced by phenylalanine. This result is consistent with that observed in Petunia hybrida [31] and other plants. The substitution of tryptophan residue in R3 may contribute to the identification of new target genes and may also result in the loss of DNA binding activity to target genes [27]. In addition, the conserved motif analysis on CeMYB protein sequences revealed that closely related CeMYBs in the phylogenetic tree were composed of similar conserved motifs. The majority of CeMYBs contained motif 1, 2, 3, and 5. Most CeMYBs ( 49\%) contained two introns and three exons. Only members of the S1 subfamily had motif 13, implying CeMYBs in S1 may have special functions that are different from other subgroups. Overall, the number of motifs, introns, and exons in the same clade were similar, while variations were found in a few clades.

Gene duplication plays an important role in plant evolution and is a main mechanism of gene family expansion. Three whole-genome duplication (WGD) events have occurred in A. thaliana [32], and C. ensifolium has experienced two WGD events [33]. The result showed that segmental and tandem duplication events occured unevenly on chromosomes. There were 23 pairs of tandemly duplicated CeMYBs and 29 pairs of segmentally duplicated CeMYBs in the $C$. ensifolium genome, suggesting that gene duplication plays an important role in the evolution of the MYB gene family in C. ensifolium. The Ka/Ks ratios of $46 C e M Y B s$ replications suggested that this gene family undergo purifying selection and highly conserved evolution. Chromosomal localization analysis failed to locate CeMYB135 and CeMYB136, which may be due to the incomplete sequence of the C. ensifolium genome or the high degree of heterozygosity. The cis-acting elements in the promoter region are involved in the transcriptional regulation of the dynamic network of gene activity, which control various biological processes and play an important role in regulating gene expression [34]. The data showed that most of the promoter regions of CeMYBs contain cis-acting elements related to resistance and are hormone responsive, with $92.6 \%$ of $C e M Y B$ promoters containing MeJA-responsive elements (CGTCA motif or TGACG motif). Moreover, the $C e M Y B$ family members also contain other cis-acting elements including flavonoid biosynthetic regulation (MBSI), salicylic acid-responsive element (TCA-element and SARE), low temperature-responsive element (LTR), and so on. This indicates that the expression of CeMYBs may be regulated by a variety of factors and especially plays active roles in the stress-resistance process. Therefore, the study of cis-acting elements in CeMYB promoters is of significant value for further research.

This work analyzed the expression patterns of CeMYB52 and CeMYB104 in S6 at different color sepals of $C$. ensifolium. The results of qRT-PCR showed that CeMYB52 and CeMYB104 were both expressed in purple-red sepals and red sepals at a higher level, and their expression levels were consistent with the content of anthocyanins in the sepals. The differential expression levels of these genes may affect ABP and resulted in different pigment patterns in the sepals. The finding screened out potential genes, which can improve the efficiency of molecular breeding, contribute to the development of new colored varieties of orchids in the future, and provide significant value for comprehension of the role of R2R3-MYB transcription factors in flower color polymorphisms. However, the mechanism of the executive function in potential genes is still unclear. We next intend to verify these gene functions through transgene studies and protein interactions, amongst other methods.

\section{Materials and Methods}

\subsection{Plant Materials}

C. ensifolium 'Mo bao' with purple-red sepals, 'Shi zhang hong' with red sepals, 'Da feng su' with yellow-green sepals, and 'Ma jiang zhi yu' with white sepals were used in this study (Figure 9). The wild plants were collected at the National Orchid Germplasm 
Resources of Fujian Agriculture and Forestry University $\left(119^{\circ} 18^{\prime}\right.$ E, $\left.26^{\circ} 05^{\prime} \mathrm{N}\right)$, Fuzhou, Fujian Province, China. All sepals were sampled and frozen in liquid nitrogen immediately and stored at $-80^{\circ} \mathrm{C}$. Three biological replicates were used for all samples.
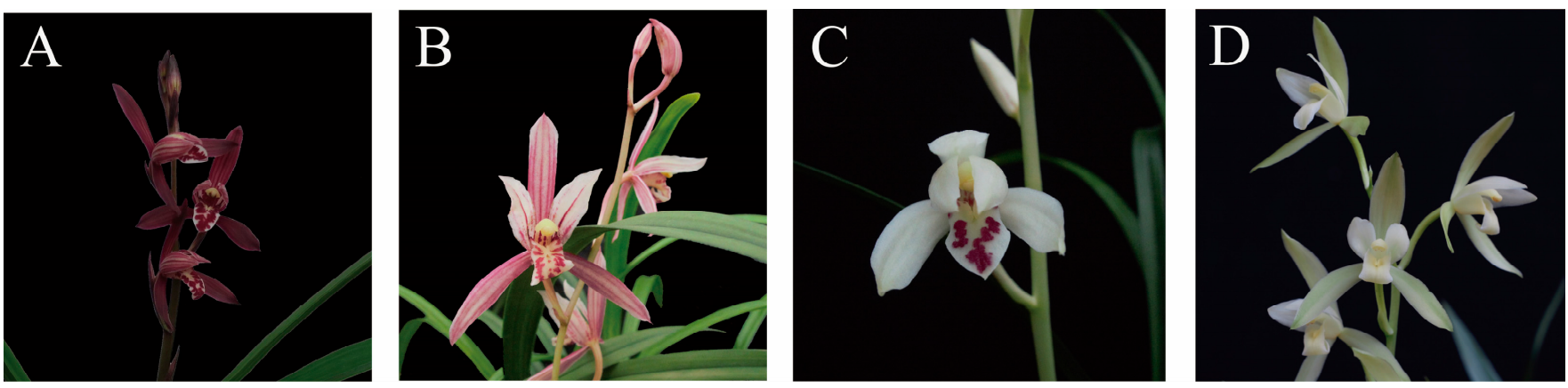

Figure 9. Sepal colors of C. ensifolium which were collected at the National Orchid Germplasm Resources of Fujian Agriculture and Forestry University in 2018. (A) The purple-red sepal. (B) The red sepal. (C) The white sepal. (D) The yellow-green sepal.

\subsection{Transcriptome Sequencing}

Sepals were collected from C. ensifolium 'Mo bao', 'Shi zhang hong', 'Da feng su', and 'Ma jiang zhi yu' for RNA extraction by using the OMEGA kit (Norcross, Georgia, USA). RNA extraction was conducted based on the manufacturer's protocol. The Illumina RNA-Seq library was constructed using an Illumina HiSeq 2500 platform by the Novogene Bioinformatics Co., Ltd. (Beijing, China). Gene expression levels were first estimated by using TopHat to map the clean reads of each sample onto the assembled genome. The obtained read counts for each gene were then normalized to FPKM (Fragments Per Kilobase of exon model per Million mapped fragments) reads. FPKM values representing the expression levels of the genes were used to generate a heat map by TBtools.

\subsection{Identification and Sequence Analysis of CeMYBs}

To identify potential CeMYBs in C. ensifolium, 131 Arabidopsis MYB protein sequences were downloaded from the Arabidopsis Information Resource (TAIR, https://www. arabidopsis.org/, accessed on 3 July 2021). BLASTP searches were performed using AtMYB amino acid sequences as queries against the $C$. ensifolium genome database. Meanwhile, the hidden Markov model (HMM) file of the MYB DNA-binding domain (PF00249) from the Pfam database (http:/ / pfam.xfam.org/search, accessed on 3 July 2021) was used to further identify CeMYB transcription factors by Simple HMM Search of TBtools. All candidate CeMYB sequences were confirmed with the NCBI conserved domain database (https: / / www. ncbi.nlm.nih.gov/Structure/cdd/wrpsb.cgi, accessed on 6 July 2021) and SMART program (http:// smart.embl-heidelberg.de/, accessed on 6 July 2021). The redundant CeMYBs were removed manually. The molecular weight and isoelectric point of CeMYB proteins were obtained through the ExPASy server (http://web.expasy.org/compute_pi/, accessed on 9 July 2021), and WoLF PSORT (https://wolfpsort.hgc.jp/, accessed on 9 July 2021) was used to predict subcellular localization [35]. After the multiple sequence alignment was generated by Clustal $\mathrm{W}$, the features of the R2 and R3 domains were identified by the online tool WebLogo (http:/ / weblogo.berkeley.edu/logo.cgi, accessed on 9 July 2021) [36].

\subsection{Phylogenetic Analysis of CeMYBs}

The full-length amino acid sequences of MYB proteins from C. ensifolium and Arabidopsis were aligned by the MUSCLE software. To construct an ML phylogenetic tree of MYBs, the aligned protein sequences of AtMYBs and CeMYBs were submitted to IQ-TREE v1.6.12 with 1000 bootstrap replicates. For better visualization, the generated ML phylogenetic tree was modified by iTOL [37]. 


\subsection{Gene Structure and Conserved Motif Analysis of CeMYBs}

The Gene Structure Display Server (GSDS, http:/ /gsds.cbi.pku.edu.cn/, accessed on 13 July 2021) was used to identify the exon-intron structure of CeMYBs [38], and Multiple Em for Motif Elicitation (MEME) (http://memesuite.org/tools/meme, accessed on 13 July 2021) [39] was used to confirm conserved motifs in CeMYB protein sequences. The parameters of MEME were as follows: the maximum number of motifs at 15, while the other parameters were kept at default. The results were visualized by TBtools software [40].

\subsection{Analysis of CeMYB Promoter Sequences}

The cis-acting elements were predicted through PlantCARE (http:/ / bioinformatics. psb.ugent.be/webtools / plantcare/html/, accessed on 13 July 2021) based on the promoter sequences ( $2 \mathrm{~kb}$ sequence upstream of the start codon) of CeMYBs [41]. Excel and TBtools were used for statistical analysis and data visualization, respectively.

\subsection{Chromosomal Localization and Synteny Analysis of CeMYBs}

The chromosomal localization of CeMYBs in the C. ensifolium genome was obtained by TBtools according to the annotation data of the $C$. ensifolium genome. The syntenic relationship between each pair of $C$. ensifolium chromosomes was displayed using One Step MCScanx program of TBtools [40]. The duplication pattern of CeMYBs was visualized by the Advance Circos package of TBtools. Then, the $K a, K s$, and $K a / K s$ values were calculated using TBtools software.

\section{8. $q R T-P C R$}

RNA from four colors sepals were reverse transcribed into cDNA using the Reverse Transcript Kit PrimerScript ${ }^{\circledR}$ RT reagent Kit with gDNA Eraser (TaKaRa, Dalian, China). The CeMYBs related to anthocyanin synthesis were screened and verified by qRT-PCR. The GAPDH gene was used as the internal reference. Primer Premier 5.0 was used to design primers (Supplementary Materials Table S5), and the relative gene expression was calculated by the $2^{-\Delta \Delta C t}$ method. The TaKaRa TB Green ${ }^{\mathrm{TM}}$ Premix Ex Taq ${ }^{\mathrm{TM}}$ II (RR820A) kit was used for qRT-PCR analysis on an ABI 7500 Real-Time System (Applied Biosystems, Foster City, CA, USA). The experimental template was a 96-well plate, and a $20 \mu \mathrm{L}$ reaction system was established per well.

\section{Conclusions}

This study identified 136 CeMYBs across the C. ensifolium genome, and analysis on phylogeny, gene structure, motif composition, chromosomal localization, and gene expression of $C e M Y B s$ were carried out. Moreover, the expression patterns of some CeMYBs were analyzed based on the transcriptome data. In short, these results provide information for further analysis of the function of $C e M Y B s$ and clarification of their biological roles. Our findings will be helpful for further research on flower color polymorphisms and the breeding of novel varieties with respect to flower color.

Supplementary Materials: The following are available online at https:/ / www.mdpi.com/article/10 .3390/ijms222413245/s1.

Author Contributions: Y.A. and Z.-J.L. conceived and designed the research. Y.-J.K. and Q.-D.Z. performed the transcriptome and gene expression analysis and wrote the original draft. Y.-H.Y. and Y.O. helped with the sampling and field work. J.-Y.C. and M.-J.W. helped to perform the experiment. H.-P.L. and L.Y. revised the manuscript. All authors contributed to revising the manuscript. Y.-J.K. and Q.-D.Z. contributed equally. All authors have read and agreed to the published version of the manuscript.

Funding: This work was supported by The National Key Research and Development Program of China (2019YFD1001003), the Fujian Natural Science Foundation Project of China (2020J01585), the Outstanding Young Scientific Research Talent Project of Fujian Agriculture and Forestry University (no. xjq201910). 
Institutional Review Board Statement: Not applicable.

Informed Consent Statement: Not applicable.

Data Availability Statement: The genomic data of C. ensifolium are openly available in the National Genomics Data Center (NGDC) at doi: 10.1038/s41438-021-00683-z. RNA-Seq data can be found with accession number PRJNA771426. The RNA-Seq data is publicly available on National Center for Biotechnology Information. The other data presented in this study are available in Supplementary Materials.

Conflicts of Interest: The authors declare no conflict of interest.

\section{References}

1. Riechmann, J.L.; Heard, J.; Martin, G.; Reuber, L.; Jiang, C.; Keddie, J.; Adam, L.; Pineda, O.; Ratcliffe, O.J.; Samaha, R.R.; et al. Arabidopsis transcription factors: Genome-wide comparative analysis among eukaryotes. Science 2000, 290, 2105-2110. [CrossRef]

2. Dubos, C.; Stracke, R.; Grotewold, E.; Weisshaar, B.; Martin, C.; Lepiniec, L. MYB transcription factors in Arabidopsis. Trends Plant Sci. 2010, 15, 573-581. [CrossRef]

3. Ogata, K.; Morikawa, S.; Nakamura, H.; Sekikawa, A.; Inoue, T.; Kanai, H.; Saral, A.; Ishll, S.; Nishimura, Y. Solution structure of a specific DNA complex of the Myb DNA-binding domain with cooperative recognition helices. Cell 1994, 79, 639-648. [CrossRef]

4. Feller, A.; Machemer, K.; Braun, E.L.; Grotewold, E. Evolutionary and comparative analysis of MYB and bHLH plant transcription factors. Plant J. 2011, 66, 94-116. [CrossRef] [PubMed]

5. Ma, D.; Constabel, C.P. MYB repressors as regulators of phenylpropanoid metabolism in plants. Trends Plant Sci. 2019, 24, 275-289. [CrossRef] [PubMed]

6. Chen, Y.; Yang, X.; He, K.; Liu, M.; Li, J.; Gao, Z.; Lin, Z.; Zhang, Y.; Wang, X.; Qiu, X.; et al. The MYB transcription factor superfamily of arabidopsis: Expression analysis and phylogenetic comparison with the rice MYB family. Plant Mol. Biol. 2006, 60, 107-124.

7. Baldoni, E.; Genga, A.; Cominelli, E. Plant MYB Transcription Factors: Their Role in Drought Response Mechanisms. Int. J. Mol. Sci. 2015, 16, 15811-15851. [CrossRef]

8. Chen, C.; Zhang, K.; Khurshid, M.; Li, J.; He, M.; Georgiev, M.I.; Zhang, X.; Zhou, M. MYB Transcription Repressors Regulate Plant Secondary Metabolism. Crit. Rev. Plant Sci. 2019, 38, 159-170. [CrossRef]

9. Zhou, M.; Zhang, K.; Sun, Z.; Yan, M.; Chen, C.; Zhang, X.; Tang, Y.; Wu, Y. LNK1 and LNK2 corepressors interact with the MYB3 transcription factor in phenylpropanoid biosynthesis. Plant Physiol. 2017, 174, 1348-1358. [CrossRef]

10. Wang, N.; Xu, H.; Jiang, S.; Zhang, Z.; Lu, N.; Qiu, H.; Qu, C.; Wang, Y.; Wu, S.; Chen, X. MYB12 and MYB22 play essential roles in proanthocyanidin and flavonol synthesis in red-fleshed apple (Malus sieversii f. niedzwetzkyana). Plant J. 2017, 90, $276-292$. [CrossRef]

11. Rausher, M.D.; Miller, R.E.; Tiffin, P. Patterns of evolutionary rate variation among genes of the anthocyanin biosynthetic pathway. Mol. Biol. Evol. 1999, 16, 266-274. [CrossRef]

12. Li, X.; Qian, X.; L ̌̈ , X.; Wang, X.; Ji, N.; Zhang, M.; Ren, M. Upregulated structural and regulatory genes involved in anthocyanin biosynthesis for coloration of purple grains during the middle and late grain-filling stages. Plant Physiol. Biochem. 2018, 130, 235-247. [CrossRef]

13. Chen, L.; Hu, B.; Qin, Y.; Hu, G.; Zhao, J. Advance of the negative regulation of anthocyanin biosynthesis by MYB transcription factors. Plant Physiol. Biochem. 2019, 136, 178-187. [CrossRef] [PubMed]

14. Ma, H.; Pooler, M.; Griesbach, R. Anthocyanin regulatory/structural gene expression in Phalaenopsis. J. Am. Soc. Hortic. Sci. 2009, 134, 88-96. [CrossRef]

15. Chiou, C.Y.; Yeh, K.W. Differential expression of MYB gene (OgMYB1) determines color patterning in floral tissue of Oncidium Gower Ramsey. Plant Mol. Biol. 2008, 66, 379-388. [CrossRef] [PubMed]

16. Li, B.J.; Zheng, B.Q.; Wang, J.Y.; Tsai, W.C.; Lu, H.C.; Zou, L.H.; Wan, X.; Zhang, D.Y.; Qiao, H.J.; Liu, Z.J.; et al. New insight into the molecular mechanism of colour differentiation among floral segments in orchids. Commun. Biol. 2020, 3, 89. [CrossRef]

17. Hsu, C.C.; Chen, Y.Y.; Tsai, W.C.; Chen, W.H.; Chen, H.H. Three R2R3-MYB transcription factors regulate distinct floral pigmentation patterning in Phalaenopsis spp. Plant Physiol. 2015, 168, 175-191. [CrossRef]

18. Fu, Z.; Wang, L.; Shang, H.; Dong, X.; Jiang, H.; Zhang, J.; Wang, H.; Li, Y.; Yuan, X.; Meng, S.; et al. An R3-MYB gene of Phalaenopsis, MYBX1, represses anthocyanin accumulation. Plant Growth Regul. 2019, 88, 129-138. [CrossRef]

19. Zhang, Y.; Zhou, T.; Dai, Z.; Dai, X.; Li, W.; Cao, M.; Li, C.; Tsai, W.C.; Wu, X.; Zhai, J.; et al. Comparative transcriptomics provides insight into floral color polymorphism in a Pleione limprichtii orchid population. Int. J. Mol. Sci. 2020, 21, 247. [CrossRef]

20. Ding, L. The Relative Expression and the Analysis of Key Genes Associated with the Formation of Floral Color and Scent in Phalaenopsis Type Dendrobium. Master's Thesis, Hainan University, Hainan, China, 2016. (In Chinese).

21. Cannon, S.B.; Mitra, A.; Baumgarten, A.; Young, N.D.; May, G. The roles of segmental and tandem gene duplication in the evolution of large gene families in Arabidopsis thaliana. BMC Plant Biol. 2004, 4, 10. [CrossRef]

22. Ke, Y.; Chen, M.; Ma, S.; Ou, Y.; Wang, Y.; Zheng, Q.; Liu, Z.; Ai, Y. Research progress of MYB transcription factors in Orchidaceae. Acta Hortic. Sin. 2021, 48, 2311-2320. (In Chinese) 
23. Wang, X.; Liang, L.; Li, L.; Wang, T. Genome-wide analysis of R2R3-MYB transcription factors in Phalaenopsis equestris. For. Res. 2018, 31, 104-113. (In Chinese)

24. Fan, H.; Cui, M.; Li, N.; Li, X.; Liang, Y.; Liu, L.; Cai, Y.; Lin, Y. Genome-wide identification and expression analyses of R2R3-MYB transcription factor genes from two Orchid species. PeerJ 2020, 8, e9781. [CrossRef]

25. Du, H.; Yang, S.S.; Liang, Z.; Feng, B.R.; Liu, L.; Huang, Y.B.; Tang, Y.X. Genome-wide analysis of the MYB transcription factor superfamily in soybean. BMC Plant Biol. 2012, 12, 106. [CrossRef] [PubMed]

26. Wei, Q.; Chen, R.; Wei, X.; Liu, Y.; Zhao, S.; Yin, X.; Xie, T. Genome-wide identification of R2R3-MYB family in wheat and functional characteristics of the abiotic stress responsive gene TaMYB344. BMC Genom. 2020, 21, 792. [CrossRef] [PubMed]

27. Ju, L.; Lei, X.; Zhao, C.; Shu, H.; Wang, Z.; Cheng, S. Identification of MYB family genes and its relationship with pungency of pepper. Acta Hortic. Sin. 2020, 47, 875-892.

28. Gigolashvili, T.; Engqvist, M.; Yatusevich, R.; Müller, C.; Flügge, U. HAG2/MYB76 and HAG3/MYB29 exert a specific and coordinated control on the regulation of aliphatic glucosinolate biosynthesis in Arabidopsis thaliana. New Phytol. 2008, 177, 627-642. [CrossRef] [PubMed]

29. Kang, Y.H.; Kirik, V.; Hulskamp, M.; Nam, K.H.; Hagely, K.; Lee, M.M.; Schiefelbein, J. The MYB23 gene provides a positive feedback loop for cell fate specification in the Arabidopsis root epidermis. Plant Cell 2009, 21, 1080-1094. [CrossRef] [PubMed]

30. Zhang, T.; Cui, Z.; Li, Y.; Kang, Y.; Song, X.; Wang, J.; Zhou, Y. Genome-Wide identification and expression analysis of MYB transcription factor superfamily in Dendrobium catenatum. Front. Genet. 2021, 12, 714696. [CrossRef]

31. Chen, G.; He, W.; Guo, X.; Pan, J. Genome-wide identification, classification and expression analysis of the MYB transcription factor family in Petunia. Int. J. Mol. Sci. 2021, 22, 4838. [CrossRef]

32. Bowers, J.E.; Chapman, B.A.; Rong, J.; Paterson, A.H. Unravelling angiosperm genome evolution by phylogenetic analysis of chromosomal duplication events. Nature 2003, 422, 433-438. [CrossRef] [PubMed]

33. Ai, Y.; Li, Z.; Sun, W.H.; Chen, J.; Zhang, D.; Ma, L.; Zhang, Q.H.; Chen, M.K.; Zheng, Q.D.; Liu, J.F.; et al. The Cymbidium genome reveals the evolution of unique morpholog traits. Hortic. Res. 2021, 8, 255. [CrossRef]

34. Yamaguchi-Shinozaki, K.; Shinozaki, K. Organization of cis-acting regulatory elements in osmotic- and cold-stress-responsive promoters. Trends Plant Sci. 2005, 10, 88-94. [CrossRef] [PubMed]

35. Horton, P.; Park, K.-J.; Obayashi, T.; Fujita, N.; Harada, H.; Adams-Collier, C.J.; Nakai, K. WoLF PSORT: Protein localization predictor. Nucleic Acids Res. 2007, 35, W585-W587. [CrossRef]

36. Crooks, G.E.; Hon, G.; Chandonia, J.M.; Brenner, S.E. WebLogo: A Sequence Logo Generator. Genome Res. 2004, 14, 1188-1190. [CrossRef]

37. Letunic, I.; Bork, P. Interactive Tree of Life (iTOL): An online tool for phylogenetic tree display and annotation. Bioinformatics 2007, 23, 127-128. [CrossRef]

38. Hu, B.; Jin, J.; Guo, A.Y.; Zhang, H.; Luo, J.; Gao, G. GSDS 2.0: An upgraded gene feature visualization server. Bioinformatics 2015, 31, 1296-1297. [CrossRef] [PubMed]

39. Bailey, T.L.; Williams, N.; Misleh, C.; Li, W.W. MEME: Discovering and analyzing DNA and protein sequence motifs. Nucleic Acids Res. 2006, 34, W369-W373. [CrossRef]

40. Chen, C.; Chen, H.; Zhang, Y.; Thomas, H.R.; Frank, M.H.; He, Y.; Xia, R. TBtools: An integrative toolkit developed for interactive analyses of big biological data. Mol. Plant 2020, 13, 1194-1202. [CrossRef]

41. Lescot, M.; Déhais, P.; Thijs, G.; Marchal, K.; Moreau, Y.; De Peer, Y.V.; Rouzé, P.; Rombauts, S. PlantCARE, a database of plant cis-acting regulatory elements and a portal to tools for in silico analysis of promoter sequences. Nucleic Acids Res. 2002, 30, 325-327. [CrossRef] 